J. Ralston and L. Sario

Nagoya Math. J.

Vol. 61 (1976), 59-71

\title{
A RELATION BETWEEN BIHARMONIC GREEN'S FUNCTIONS OF SIMPLY SUPPORTED AND CLAMPED BODIES
}

\author{
JAMES RALSTON AND LEO SARIO
}

The deflection, under a point load, of a thin elastic plate clamped at the edges is the biharmonic Green's function $\beta$ with the boundary data $\beta=\partial \beta / \partial n=0$. If the boundary of the region is reasonably smooth, the construction of $\beta$ offers no difficulty. In contrast, nothing is known about the existence of $\beta$ in the general case. The purpose of our study is to give a sufficient condition for the existence of $\beta$ on a given Riemannian manifold of arbitrary dimension and to construct $\beta$. Our results will have, apart from their physical meaning in elasticity, consequences in the biharmonic classification theory of Riemannian manifolds.

To understand the nature of the problem, we recall that the deflection, under a point load, of a simply supported thin elastic plate is the biharmonic Green's function $\gamma$ which satisfies the boundary conditions $\gamma=\Delta \gamma=0$. The function $\gamma$ is always positive and increases with the region. As a consequence, $\gamma$ on an arbitrary plate or, more generally, on an arbitrary Riemannian manifold $R$, can be defined as the directed limit, if it is finite, of the corresponding functions $\gamma_{\Omega}$ on regular subregions $\Omega$ exhausting $R$ (Sario [34]).

Hadamard conjectured in his 1908 prize memoir [14] that the deflection $\beta$, under a point load, of a clamped plate as well be always positive. However, this conjecture was disproved by Duffin [8] for an infinite strip, by Loewner [19] and Szegö [52] for certain nonconvex plane regions, and by Garabedian [10] for a sufficiently eccentric ellipse. Interest in the problem was recently revived by the address of Duffin [9]

Received April 30, 1975.

The work was sponsored by the National Science Foundation, Grant GP 38519, and the U.S. Army Research Office, Grant DA-ARO-37-124-73-G39, University of California, Los Angeles.

MOS Classification 31B30. 
delivered before the 1974 Annual Meeting of the American Mathematical Society in San Francisco. The possibility of a nonconstant sign of $\beta_{\Omega}$ on regular subregions $\Omega$ of $R$ causes difficulty in the convergence proof as $\Omega \rightarrow R$. This is where the problem lies in defining and constructing $\beta$.

To gain intuition from a concrete case we first consider the Euclidean $N$-space $E^{N}$. On exhausting balls $\Omega=\{r<\rho\}, \rho \rightarrow \infty$, the functions $\gamma_{\Omega}, \beta_{\Omega}$ with poles at the origin can be explicitly constructed. It turns out that $\gamma_{\Omega} \rightarrow \infty$ and $\beta_{\Omega} \rightarrow \infty$ at every point if $N \leq 4$, whereas both $\gamma$ $=\lim _{\Omega} \gamma_{\Omega}$ and $\beta=\lim _{\Omega} \beta_{\Omega}$ exist on $E^{N}$ if $N>4$. This simultaneous existence of $\gamma$ and $\beta$ raises the question: is there some relationship between the existence of $\gamma$ and $\beta$ in the general case (and, in particular, on a plate of arbitrary shape)? That the answer is in the affirmative is our main result: on an arbitrarily given Riemannian manifold, if $\gamma$ exists, so does $\beta$. The interesting problem whether or not the converse is true is not a topic of the present study.

A necessary and sufficient condition is known for the existence of $\gamma$ : the harmonic measure $\omega$ of the ideal boundary of the manifold, with values 1 on a compact set, has to be in $L^{2}$ (Sario [34]). This test now carries over as a sufficient condition for the existence of $\beta$. As an example, the above result on the existence of $\beta$ on $E^{N}$ for $N>4$ follows immediately, and is independent of the pole and exhaustion. Similarly, $\beta$ is seen to exist on the Poincare $N$-ball $B^{N}=\{r<1\}$ with the metric $d s=\left(1-r^{2}\right)^{\alpha}|d x|, \alpha$ a constant, if $\alpha>-3 / 2$ for $N=2, \alpha \in(-3,1)$ for $N=3$, and $\alpha<(N-2)^{-1}$ for $N>3$.

A comprehensive bibliography, which includes recent work in the field, is attached.

1. To construct the biharmonic Green's function $\gamma$ on the Euclidean $N$-space $E^{N}$, we exhaust $E^{N}$ by $N$-balls $\Omega=\{r<\rho\}, \rho \rightarrow \infty$. We recall that the biharmonic fundamental singularity $\sigma$ at $r=0$ is $r^{2} \log r$ for $N=2, r$ for $N=3, \log r$ for $N=4$, and $r^{-N+4}$ for $N>4$, the singularity manifesting itself in that $\sigma \notin C^{4}$ at the origin. We normalize $\sigma$ to vanish for $r=\rho$ and then subtract a multiple of the regular biharmonic function $r^{2}-\rho^{2}$ such that the Laplace-Beltrami operator $\Delta=d \delta+\delta d$ acting on $\gamma_{\Omega}$,

$$
\Delta \gamma_{\Omega}=-\frac{1}{\sqrt{g}} \frac{\partial}{\partial r}\left(\sqrt{g} \frac{\partial}{\partial r} \gamma_{\Omega}\right)
$$


gives zero values for $r=\rho$. The resulting $\gamma_{\Omega}$ on $\bar{\Omega}$ with pole 0 is for $N=2,3,4,>4$, respectively,

$$
\gamma_{\Omega}=\left\{\begin{array}{l}
r^{2} \log \frac{r}{\rho}-\left(r^{2}-\rho^{2}\right), \\
-r+\rho+\frac{1}{3} \rho^{-1}\left(r^{2}-\rho^{2}\right), \\
-\log \frac{r}{\rho}+\frac{1}{4} \rho^{-2}\left(r^{2}-\rho^{2}\right), \\
r^{-N+4}-\rho^{-N+4}+N^{-1}(N-4) \rho^{-N+2}\left(r^{2}-\rho^{2}\right)
\end{array}\right.
$$

We define $\gamma$ on $E^{N}$ as $\gamma=\lim _{\rho \rightarrow \infty} \gamma_{s}$ and conclude that $\gamma$ with pole 0 does not exist on $E^{N}$ for $N=2,3,4$, whereas for $N>4$ it is

$$
\gamma=r^{-N+4}
$$

For $\beta_{\Omega}$ on $\Omega$ the construction is the same except that now a multiple of $r^{2}-\rho^{2}$ is to be subtracted which makes $\partial \beta_{\Omega} / \partial n=0$ for $r=\rho$. We obtain for $N=2,3,4,>4$,

$$
\beta_{\Omega}=\left\{\begin{array}{l}
r^{2} \log \frac{r}{\rho}-\frac{1}{2}\left(r^{2}-\rho^{2}\right) \\
-r+\rho+\frac{1}{2} \rho^{-1}\left(r^{2}-\rho^{2}\right) \\
-\log \frac{r}{\rho}+\frac{1}{2} \rho^{-1}\left(r^{2}-\rho^{2}\right) \\
r^{-N+4}-\rho^{N+4}+\frac{1}{2}(N-4) \rho^{-N+2}\left(r^{2}-\rho^{2}\right) .
\end{array}\right.
$$

As $\rho \rightarrow \infty$, we again deduce that $\beta=\lim _{\rho \rightarrow \infty} \beta_{\Omega}$ does not exist on $E^{N}$ for $N \leq 4$, whereas for $N>4$ it is

$$
\beta=r^{-N+4} .
$$

That for the singularity $y \in E^{N}$, the existence of $\gamma=\gamma(x, y)$ and $\beta=\beta(x, y)$ for $N>4$ is independent of $(x, y)$ and the exhaustion will be seen in No. 9.

The biharmonic Green's functions $\gamma$ and $\beta$ exist on $E^{N}$ for precisely the same dimensions: $N>4$.

This observation suggests an inquiry into a possible relationship between the existence of $\gamma$ and $\beta$ on every Riemannian manifold. 
2. Given an arbitrary Riemannian manifold $R$, let $\Omega$ be a regular subregion of $R$. Take a point $y \in \Omega$ and a small geodesic ball $B_{y}$ about $y, \alpha_{y}=\partial \beta_{y}, \alpha_{y} \cap \partial \Omega=\emptyset$, and orient both $\alpha_{y}$ and $\partial \Omega$ positively with respect to $B_{y}$ and $\Omega$, respectively. Let $\beta_{\Omega}=\beta_{\Omega}(x, y), \gamma_{\Omega}=\gamma_{\Omega}(x, y)$ be the biharmonic Green's functions on $\bar{\Omega}$, with the biharmonic fundamental singularity at $y$ normalized by $\int_{\alpha_{y}} * d \Delta \beta_{\Omega}=\int_{\alpha_{y}} * d \Delta \gamma_{\Omega}=-1$, and with boundary data

$$
\beta_{\Omega}=\frac{\partial \beta_{\Omega}}{\partial n}=\gamma_{\Omega}=\Delta \gamma_{\Omega}=0 \quad \text { on } \partial \Omega
$$

For $f \in C_{0}^{\infty}(\Omega)$, set

$$
\left(\beta_{\Omega} f\right)(x)=\int_{\Omega} \beta_{\Omega}(x, y) * f(y), \quad\left(\gamma_{\Omega} f\right)(x)=\int_{\Omega} \gamma_{\Omega}(x, y) * f(y) .
$$

By general results on elliptic boundary value problems (e.g., Hörmander $[16$, Ch. $X])$, there are unique functions $u_{\beta}, u_{r} \in C^{\infty}(\bar{\Omega})$ satisfying

$$
\begin{gathered}
\Delta^{2} u_{\beta}=\Delta^{2} u_{r}=f \quad \text { on } \Omega, \\
u_{\beta}=\frac{\partial u_{\beta}}{\partial n}=u_{r}=\Delta u_{r}=0 \quad \text { on } \partial \Omega .
\end{gathered}
$$

For every $u_{\beta}, u_{r}$ with these properties,

$$
u_{\beta}=\beta_{\Omega} f, \quad u_{r}=\gamma_{\Omega} f .
$$

In fact, by Stokes' formula

$$
\begin{aligned}
\int_{\partial \Omega-\alpha_{y}} & u_{\beta} * d \Delta \beta_{\Omega}-\Delta \beta_{\Omega} * d u_{\beta}-\beta_{\Omega} * d \Delta u_{\beta}+\Delta u_{\beta} * d \beta_{\Omega} \\
= & -\int_{\Omega-B_{y}} u_{\beta} * \Delta^{2} \beta_{\Omega}-\Delta \beta_{\Omega} * \Delta u_{\beta}-\beta_{\Omega} * f+\Delta u_{\beta} * \Delta \beta_{\Omega} .
\end{aligned}
$$

As the geodesic radius of $\alpha_{y}$ tends to $0, \int_{-\alpha_{y}} \rightarrow u_{\beta}(y)$, and we obtain $u_{\beta}=\beta_{\Omega} f$. Similarly, the equality

$$
\begin{aligned}
\int_{\partial \Omega-\alpha_{y}} u_{r} * d \Delta \gamma_{\Omega}-\Delta \gamma_{\Omega} * d u_{r}-\gamma_{\Omega} * d \Delta u_{r}+\Delta u_{r} * d \gamma_{\Omega} \\
\quad=-\int_{\Omega-B_{y}} u_{r} * \Delta^{2} \gamma_{\Omega}-\Delta \gamma_{\Omega} * \Delta u_{r}-\gamma_{\Omega} * f+\Delta u_{r} * \Delta \gamma_{\Omega}
\end{aligned}
$$

gives $u_{r}=\gamma_{0} f$. 
3. Let $\Omega, \Omega^{\prime}$ with $\Omega \subset \Omega^{\prime}$ be regular subregions of $R$, and set $(f, g)$ $=\int_{\Omega} f * g$.

LEMma 1. For $f \in C_{0}^{\infty}(\Omega)$,

$$
\left(f, \beta_{\Omega} f\right) \leq\left(f, \beta_{\Omega^{\prime}} f\right) .
$$

Proof. On $\Omega$, consider the Sobolev space

$$
H_{2, \beta}(\Omega)=\left\{u\left|D^{\alpha} u \in L^{2}(\Omega),\right| \alpha \mid \leq 2, u=\frac{\partial u}{\partial n}=0 \text { on } \partial \Omega\right\}
$$

and the functional on $H_{2, \beta}(\Omega)$,

$$
J(u)=\int_{\Omega} \frac{1}{2} \Delta u * \Delta u-u * f .
$$

For $u \in H_{2, \beta}$ one has the elliptic estimate for $|\alpha| \leq 2$,

$$
\left\|D^{\alpha} u\right\|^{\Omega} \leq C_{\alpha}\|\Delta u\|^{\Omega},
$$

where $\|\cdot\|^{\Omega}$ stands for the $L^{2}$ norm over $\Omega$. Thus $J(u)$ is bounded below. Let $\left\{u_{n}\right\}$ be a sequence such that $J\left(u_{n}\right) \rightarrow \inf _{H_{2, \beta}} J(u)$. Then $\left\{\Delta u_{n}\right\}$ is a bounded sequence in $L^{2}(\Omega)$. Using (1) and the Rellich compactness theorem, we may choose a subsequence $\left\{u_{n_{k}}\right\}$ converging to $\bar{u}$ in $L^{2}(\Omega)$ and converging weakly to $\bar{u}$ in $H_{2}(\Omega)$. Since $H_{2, \beta}$ is a closed subspace of $H_{2}(\Omega)$, it follows that $\bar{u} \in H_{2, \beta}$. Now $J\left(u_{n}\right) \rightarrow \inf _{H_{2, \beta}} J(u)$ implies $\lim _{k \rightarrow \infty}\left\|\Delta u_{n_{k}}\right\|=\|\Delta \bar{u}\|$. Hence, $\left\{\Delta u_{n_{k}}\right\}$ converges to $\Delta \bar{u}$ in $L^{2}(\Omega)$ and

$$
\inf _{H_{2, \beta}} J(u)=J(\bar{u}) .
$$

Thus $\bar{u}$ satisfies

$$
0=\int_{\Omega} \Delta \bar{u} * \Delta \varphi-\varphi * f
$$

for all $\varphi \in H_{2, \beta}$. By direct computation, $u_{\beta}$ also satisfies this equation. A fortiori,

$$
0=\int_{\Omega} \Delta\left(\bar{u}-u_{\beta}\right) * \Delta \varphi
$$

for all $\varphi \in H_{2, \beta}$. In particular, this holds for $\varphi=\bar{u}-u_{\beta}$ and thus (1) implies $\bar{u}=u_{\beta}$. As a consequence,

$$
\inf _{u \in H_{2, \beta}} J(u)=J\left(u_{\beta}\right)=\int_{\Omega} \frac{1}{2} \Delta u_{\beta} * \Delta u_{\beta}-u_{\beta} * \Delta^{2} u_{\beta} .
$$


An integration by parts gives

$$
J\left(u_{\beta}\right)=-\frac{1}{2} \int_{\Omega} \Delta u_{\beta} * \Delta u_{\beta} .
$$

Every $u \in H_{2, \beta}(\Omega)$ can be extended to a function $u \in H_{2, \beta}\left(\Omega^{\prime}\right)$ by setting $u=0$ on $\Omega^{\prime}-\Omega$. Therefore,

$$
\min _{H_{2, \beta}(\Omega)} J(u) \geq \min _{H_{2}, \beta\left(\Omega^{\prime}\right)} J(u),
$$

that is,

$$
\int_{\Omega} \Delta\left(\beta_{\Omega} f\right) * \Delta\left(\beta_{\Omega} f\right) \leq \int_{\Omega^{\prime}} \Delta\left(\beta_{\Omega^{\prime}} f\right) * \Delta\left(\beta_{\Omega^{\prime}} f\right)
$$

which gives

$$
\int_{\Omega} \Delta^{2}\left(\beta_{\Omega} f\right) * \beta_{\Omega} f \leq \int_{\Omega} \Delta^{2}\left(\beta_{\Omega^{\prime}} f\right) * \beta_{\Omega^{\prime}} f
$$

The Lemma follows.

4. We now compare $\beta_{\Omega}$ and $\gamma_{\Omega}$ on the same regular subregion $\Omega \subset R$.

LeMma 2. For $f \in C_{0}^{\infty}(\Omega)$,

$$
\left(f, \beta_{\Omega} f\right) \leq\left(f, \gamma_{\Omega} f\right) .
$$

Proof. For the Sobolev spaces

$$
\begin{aligned}
H_{2, r}(\Omega) & =\left\{u\left|D^{\alpha} u \in L^{2}(\Omega),\right| \alpha|\leq 2, u| \partial \Omega=0\right\}, \\
H_{2}(\Omega) & =\left\{u\left|D^{\alpha} u \in L^{2}(\Omega),\right| \alpha \mid \leq 2\right\},
\end{aligned}
$$

we have $H_{2, \beta} \subset H_{2, \gamma} \subset H_{2}$, and therefore,

$$
\min _{H_{2, \beta}(\Omega)} J(u) \geq \min _{H_{2, \gamma}(\Omega)} J(u)
$$

An argument completely analogous to that in No. 3, with $H_{2, \beta}$ and $u_{\beta}$ replaced by $H_{2, r}$ and $u_{r}$, gives

$$
J\left(u_{r}\right)=\inf _{H_{2, \gamma}(\Omega)} J(u)
$$

Then we have

$$
-\frac{1}{2} \int_{\Omega} \Delta u_{\beta} * \Delta u_{\beta}=J\left(u_{\beta}\right) \geq J\left(u_{r}\right)=-\frac{1}{2} \int_{\Omega} \Delta u_{r} * \Delta u_{r} .
$$


As a consequence,

$$
\int_{\Omega} \Delta u_{\beta} * \Delta u_{\beta} \leq \int_{\Omega} \Delta u_{\tau} * \Delta u_{\gamma} .
$$

Here

$$
\begin{gathered}
\int_{\Omega} \Delta u_{r} * \Delta u_{r}=-\int_{\partial \Omega} \Delta u_{r} * d u_{r}+\int_{\Omega} d \Delta u_{r} * d u_{r} \\
=\int_{\partial \Omega} u_{r} * d \Delta u_{r}+\int_{\Omega} u_{r} * \Delta^{2} u_{r}=\int_{\Omega} u_{r} * f,
\end{gathered}
$$

and we infer that

$$
\int_{\Omega} u_{\beta} * f \leq \int_{\Omega} u_{r} * f
$$

hence the Lemma.

5. Thus far $\Omega$ has been a fixed regular subregion of $R$. We now let $\Omega$ exhaust $R$, and indicate inner products taken over $\Omega$ by the subscript $\Omega$. We know that either $\lim _{\Omega \rightarrow R} \gamma_{\Omega}(x, y)=\infty$ for every $(x, y)$, or else the biharmonic Green's function $\gamma(x, y)=\lim _{\Omega \rightarrow R} \gamma_{\Omega}(x, y)$ on $R$ exists for every $(x, y)$, with the convergence uniform on compact sets of $R$ (Sario [34]). Let $L_{0}^{2}(R)$ be the space of $L^{2}$ functions on $R$ with compact supports.

LEMMA 3. If $\gamma$ exists on $R$, then as $\Omega \rightarrow R,\left(g, \beta_{\Omega} f\right)^{\Omega}$ converges for all $f, g \in L_{0}^{2}(R)$.

Proof. Since $C_{0}^{\infty}$ is dense in $L^{2}$, Lemmas 1 and 2 remain valid for $f \in L_{0}^{2}(R), \operatorname{supp} f \subset \Omega$. If $\gamma$ exists, the uniform convergence $\gamma_{\Omega} \rightarrow \gamma$ on compact sets entails the existence of a constant $M_{f}$ such that

$$
\left(f, \gamma_{\Omega} f\right)^{\Omega}<M_{f}
$$

for $f \in C_{0}^{\infty}(R)$ and hence for $f \in L_{0}^{2}(R)$. By Lemmas 1 and 2 for $f \in L_{0}^{2}(\Omega)$, $\left(f, \beta_{\Omega} f\right)^{\Omega}$ converges. For $f, g \in L_{0}^{2}(R)$,

$$
2\left(g, \beta_{\Omega} f\right)^{\Omega}=\left(f+g, \beta_{\Omega}(f+g)\right)^{\Omega}-\left(f, \beta_{\Omega} f\right)^{\Omega}-\left(g, \beta_{\Omega} g\right)^{\Omega}
$$

and therefore $\left(g, \beta_{\Omega} f\right)^{\Omega}$ converges.

6. To prove the convergence of $\beta_{\Omega}$ in every compact set $K$ of an $R$ which carries $\gamma$, we may assume that $y \notin K$. In fact, if $\rho(x, y)$ is the geodesic distance between $x$ and $y$, we may choose, for sufficiently small 
constants $\rho_{1}, \rho_{2}>0$,

$$
K=\left\{x \mid \rho_{1} \leq \rho(x, y) \leq \rho_{2}\right\} .
$$

The uniform convergence of $\beta_{\Omega}$ on $K$ will imply that of the harmonic function $\Delta \beta_{\Omega}$ on $K$ and hence on $K_{0}=\left\{x \mid 0 \leq \rho(x, y) \leq \rho_{2}\right\}$. A fortiori, the potential part in the Riesz decomposition of $\beta_{\Omega}$ converges uniformly on $K_{0}$ and so does, by the convergence of $\beta_{\Omega}$ on $K$, the harmonic part on $K$ and consequently on $K_{0}$.

Having chosen an arbitrary but then fixed compact set $K$ of $R$, $y \notin K$, we take a compact set $K_{y}, y \in K_{y}, K \cap K_{y}=\emptyset$, and a function $\varphi \in C_{0}^{\infty}(R), \varphi \geq 0, \varphi \mid K_{y}=1, K_{\varphi}=\operatorname{supp} \varphi$ a regular region, $K_{\varphi} \cap K=\emptyset$, and let $\Omega$ henceforth contain $K_{\varphi} \cup K$. We denote by $\beta_{\varphi}$ the biharmonic Green's function $\beta$ on $K_{\varphi}$ and set

$$
\tilde{\beta}_{\Omega}=\beta_{\Omega}-\varphi \beta_{\varphi}, \quad \Delta_{x}^{2} \tilde{\beta}_{\Omega}=f,
$$

where $f$ is independent of $\Omega$. Since $\Delta^{2}\left(\tilde{\beta}_{\Omega}-\beta_{\Omega} f\right)=0$ and $\tilde{\beta}_{\Omega}-\beta_{\Omega} f=$ $\partial\left(\tilde{\beta}_{\Omega}-\beta_{\Omega} f\right) / \partial n=0$ on $\partial \Omega$, we have

$$
\tilde{\beta}_{\Omega}=\beta_{\Omega} f \text {. }
$$

Denote the $L^{2}$ norm by $\|\cdot\|$ and choose compact sets $K_{1}, K_{2}$ with $K \subset \operatorname{int} K_{1} \subset K_{1} \subset \operatorname{int} K_{2} \subset K_{2}$ and $K_{2} \cap K_{\varphi}=\emptyset, K_{2} \subset \Omega$.

LEMMA 4. The $L^{2}$ norm $\left\|\tilde{\beta}_{\Omega}\right\|^{K_{2}}$ is bounded in $\Omega$.

Proof. For the functions $g \in L_{0}^{2}(R)$, the restrictions $g \mid K_{2}$ form a Banach space $B$, on which $\tilde{\beta}_{\Omega}$ gives a bounded linear functional $\left(g, \tilde{\beta}_{\Omega}\right)^{K_{2}}$,

$$
\left|\left(g, \tilde{\beta}_{\Omega}\right)\right|^{K_{2}} \leq k_{\Omega}\|g\|^{K_{2}}, \quad k_{\Omega}=\left\|\tilde{\beta}_{\Omega}\right\|^{K_{2}} .
$$

By the Uniform Boundedness Principle, either there exists a constant $k$ independent of $\Omega$ with

$$
\left|\left(g, \tilde{\beta}_{\Omega}\right)\right|^{K_{2}} \leq k\|g\|^{K_{2}}
$$

for all $g \in B$, or else

$$
\left|\left(g, \tilde{\beta}_{\Omega}\right)\right|^{K_{2}} \rightarrow \infty
$$

for some $g$ (in fact for $g$ in a dense set in $B$ ) as $\Omega \rightarrow R$. But we know from Lemma 3 that $\left(g, \tilde{\beta}_{\Omega}\right)=\left(g, \beta_{\Omega} f\right)$ converges for every $g$. Therefore, the first alternative occurs. In particular, for $g=\tilde{\beta}_{\Omega} \mid K_{2}$,

$$
\left(\left\|\tilde{\beta}_{\Omega}\right\|^{K_{2}}\right)^{2} \leq k\left\|\tilde{\beta}_{\Omega}\right\|^{K_{2}},
$$


hence the Lemma.

7. We are ready to state our main result:

THEOREM. On an arbitrarily given Riemannian $N$-manifold $R, N$ $\geq 2$, if the biharmonic Green's function of the simply supported body,

$$
\gamma(x, y)=\lim _{\Omega \rightarrow R} \gamma_{\Omega}(x, y)
$$

exists for some, and hence every, $(x, y)$, then so does the biharmonic Green's function of the clamped body,

$$
\beta(x, y)=\lim _{\Omega \rightarrow R} \beta_{\Omega}(x, y),
$$

and the convergence is uniform on compact sets of $R$.

Proof. By virtue of the interior regularity estimate (cf. Agmon [1, Sec. 5]) in Sobolev norms,

$$
\left\|\tilde{\beta}_{\Omega}\right\|_{s+4}^{K_{1}} \leq C\left(\|f\|_{s}^{K_{2}}+\left\|\tilde{\beta}_{\Omega}\right\|^{K_{2}}\right),
$$

Lemma 4 implies that

$$
\left\|D^{\alpha} \tilde{\beta}_{\Omega}\right\|^{K_{1}} \text { is bounded in } \Omega
$$

for every $m \geq 0,|\alpha| \leq m$. Therefore, by the Rellich compactness theorem (cf. [1, Sec. 3]), there exists a sequence $\left\{\tilde{\beta}_{n}=\tilde{\beta}_{\Omega_{n}}\right\}$ such that, for $|\alpha| \leq m$,

$$
\left\|D^{\alpha}\left(\tilde{\beta}_{n+p}-\tilde{\beta}_{n}\right)\right\|^{K_{1}} \rightarrow 0
$$

as $n \rightarrow \infty, p \geq 0$. This in turn implies by Sobolev's Inequality (cf. [1, Sec. 3]) that, for $|\alpha| \leq m$,

$$
\left|D^{\alpha}\left(\tilde{\beta}_{n+p}-\tilde{\beta}_{n}\right)\right| \rightarrow 0 .
$$

In particular, for $\alpha=0$ we have by $\tilde{\beta}_{n}\left|K=\beta_{n}\right| K$ the desired uniform convergence of $\beta_{n}$ on $K$. The uniqueness of the limiting function $\beta$ on $R$ follows from

$$
\left(g, \beta_{n} g\right) \nearrow \sup _{\{\Omega\}}\left(g, \beta_{\Omega} g\right),
$$

which implies

$$
(g, \beta g)=\sup _{\{\Omega\}}\left(g, \beta_{\Omega} g\right)
$$

for all $g \in L_{0}^{2}(R)$ with supp $g \subset K$. 
This completes the proof of the Theorem.

8. The criteria known for the existence of $\gamma$ are now available, as sufficient conditions, for the existence of $\beta$. In particular, for a fixed regular subregion $\Omega_{0}$ of $R, \bar{\Omega}_{0} \subset \Omega$, let $\omega_{\Omega}$ be the harmonic measure on $\bar{\Omega}-\Omega_{0}$ with $\omega_{\Omega}\left|\partial \Omega_{0}=1, \omega_{\Omega}\right| \partial \Omega=0$. The limiting function $\omega=\lim _{\Omega \rightarrow R} \omega_{\Omega}$ is the harmonic measure of the ideal boundary of $R$ on $R-\Omega_{0}$. It is known (e.g. Sario-Nakai [37]) that $\omega \equiv 1$ if and only if $R$ belongs to the class $\mathcal{O}_{G}$ of parabolic Riemannian manifolds, characterized by the nonexistence of the harmonic Green's function $g(x, y)=\lim _{\Omega \rightarrow R} g_{\Omega}(x, y)$. Moreover, $\gamma$ exists on $R$ if and only if $R \notin \mathcal{O}_{G}$ and $\omega \in L^{2}\left(R-\Omega_{0}\right)$ (Sario [34]). The Theorem yields:

CoRollary. If $R \notin \mathcal{O}_{G}$ and $\omega \in L^{2}\left(R-\Omega_{0}\right)$, then $\beta$ exists on $R$.

For the sake of completeness we give a (slightly simplified) proof. Choose $x, y \in R$ and then $\Omega_{0}$ with $x, y \in \Omega_{0}$. For $\Omega \supset \bar{\Omega}_{0}$ we have by virtue of $\gamma_{\Omega}=\Delta \gamma_{\Omega}=0$ on $\partial \Omega$,

$$
\gamma_{\Omega}(x, y)=\int_{\Omega} g_{\Omega}(x, z) * g_{\Omega}(z, y)=\int_{\Omega} g_{\Omega}(z, x) * g_{\Omega}(z, y) .
$$

If $R \notin \mathcal{O}_{G}$, then $g_{\Omega}(z, x) \rightarrow g(z, x), g_{\Omega}(z, y) \rightarrow g(z, y)$ on $R$, and $\omega_{\Omega} \rightarrow \omega \neq 1$ on $R-\bar{\Omega}_{0}$. There exists a compact set $C$ and constants $0<m<M<\infty$ such that both $g_{\Omega}(z, x)$ and $g_{\Omega}(z, y)$ are between $m$ and $M$ for all $z \in \partial \Omega_{0}$ and all $\Omega \supset C$. Therefore they are between $m \omega_{\Omega}$ and $M \omega_{\Omega}$ on $\partial \Omega_{0} \cup \partial \Omega$, hence on $\bar{\Omega}-\Omega_{0}$, and we have

$$
m^{2} \int_{\Omega-\Omega_{0}} \omega_{\Omega} * \omega_{\Omega}<\int_{\Omega-\Omega_{0}} g_{\Omega}(x, z) * g_{\Omega}(z, y)<M^{2} \int_{\Omega-\Omega_{0}} \omega_{\Omega} * \omega_{\Omega}
$$

If $\omega \in L^{2}\left(R-\Omega_{0}\right)$, the integral in the middle converges and so does $\gamma_{\Omega}(x, y)$. Conversely, if $\gamma_{\Omega}(x, y)$ converges, so does the integral in the middle, and $\omega \in L^{2}\left(\Omega-\Omega_{0}\right)$. The Theorem then gives the Corollary.

9. As an immediate application of the Corollary, we have the result of No. 1 that $E^{N}$ carries $\beta$ if $N>4$. Moreover, we now see that this fact is independent of the pole $y$ and the exhaustion $\{\Omega\}$ of $E^{N}$. Indeed, for $N>2, \Omega_{0}=\{r<1\}, \rho>1, \Omega=\{r<\rho\}, \rho \rightarrow \infty$,

$$
\omega_{\Omega}=\frac{r^{-N+2}-\rho^{-N+2}}{1-\rho^{-N+2}} \rightarrow r^{-N+2} .
$$


For $N>4$, we have $\|\omega\|<\infty$, hence $\beta$ exists on $E^{N}$.

For further illustration of the Corollary, consider the Poincaré $N$ ball

$$
B_{\alpha}^{N}=\left\{r<1\left|d s=\left(1-r^{2}\right)^{\alpha}\right| d x \mid\right\},
$$

$\alpha$ a constant. A function $h(r)$ on $B_{\alpha}^{N}$ is harmonic if and only if

$$
\Delta h(r)=-\frac{1}{r^{N-1}\left(1-r^{2}\right)^{N \alpha}}\left\{r^{N-1}\left(1-r^{2}\right)^{(N-2) \alpha} h^{\prime}(r)\right\}^{\prime}=0,
$$

that is,

$$
h(r)=c \int_{a}^{r} r^{-N+1}\left(1-r^{2}\right)^{-(N-2) \alpha} d r .
$$

This is bounded, hence $B_{\alpha}^{N} \notin \mathcal{O}_{G}$, for every $\alpha$ if $N=2$, for $\alpha<(N-2)^{-1}$ if $N>2$. The harmonic measure is

$$
\omega(r) \sim \begin{cases}c \log r & \text { for } N=2, \\ c(1-r)^{-(N-2) \alpha+1} & \text { for } N>2 .\end{cases}
$$

For $0<\rho_{0}<\rho<1$,

$$
\left(\|\omega\|^{\left\{\rho_{0}<r<\rho\right\}}\right)^{2} \sim \begin{cases}c(1-\rho)^{2 \alpha+3} & \text { for } N=2, \\ c(1-\rho)^{-(N-4) \alpha+3} & \text { for } N>2,\end{cases}
$$

and

$$
B_{\alpha}^{N} \text { carries } \beta \text { if } \begin{cases}\alpha>-\frac{3}{2} & \text { for } N=2, \\ -3<\alpha<1 & \text { for } N=3 \\ \alpha<\frac{1}{N-2} & \text { for } N>3 .\end{cases}
$$

\section{BIBLIOGRAPHY}

[1] S. Agmon, Lectures on Elliptic Boundary Value Problems, Van Nostrand, Princeton, $1965,291 \mathrm{pp}$.

[2] L. Chung, Manifolds carrying bounded quasiharmonic but no bounded harmonic functions Math. Scand. (to appear).

[3] - Asymptotic behavior and biharmonic degeneracy (to appear).

[4] L. Chung and L. Sario, Harmonic and quasiharmonic degeneracy of Riemannian manifolds, Tôhoku Math. J. 27, 487-496.

[ 5 ] —- Harmonic $L^{p}$ functions and quasiharmonic degeneracy, J. Indian Math. Soc. (to appear).

[6] L. Chung, L. Sario and C. Wang, Riemannian manifolds with bounded Dirichlet 
finite polyharmonic functions, Ann. Scuola Norm. Sup. Pisa 27 (1973), 1-6.

[ 7 ] - Quasiharmonic $L^{p}$ functions on Riemannian manifolds, Ann. Scuola Norm. Sup. Pisa 2 (1974), 469-478.

[ 8 ] R. J. Duffin, On a question of Hadamard concerning super-biharmonic functions, J. Math. Physics 27 (1949), 253-258.

[9] - Some problems of mathematics and science, Bull. Amer. Math. Soc. 80 (1974), 1053-1070.

[10] P. R. Garabedian, A partial differential equation arising in conformal mapping, Pacific J. Math. 1 (1951), 485-524.

[11] D. Hada, L. Sario and C. Wang, Dirichlet finite biharmonic functions on the Poincaré $N$-ball, J. Reine Angew. Math. 272 (1975), 92-101.

[12] —,$N$-manifolds carrying bounded but no Dirichlet finite harmonic functions, Math. J. 54 (1974), 1-6.

[13] — Bounded biharmonic functions on the Poincaré $N$-ball, Kōdai Math. Sem. Rep. 26 (1975), 327-342.

[14] J. Hadamard, Mémoire sur le problème d'analyse relatif à l'équilibre des plaques élastiques encastrées, Mémoires présentés par divers savants étrangers à l'Académie des Sciences, 33 (1908), 515-629.

[15] 0. Haupt, Über das asympototische Verhalten der Lösungen gewisser linearer gewöhnlicher Differentialgleichungen, Math. Z. 48 (1913), 289-292.

[16] E. Hille, Behavior of solutions of linear second order differential equations, Ark. Mat. 2 (1952), 25-41.

[17] L. Hörmander, Linear Partial Differential Operators, Springer, New York, 1969, $285 \mathrm{pp}$.

[18] Y. K. Kwon, L. Sario and B. Walsh, Behavior of biharmonic functions on Wiener's and Royden's compactifications, Ann. Inst. Fourier (Grenoble) 21 (1971), 217-226.

[19] C. Loewner, On generalization of solutions of the biharmonic equation in the plane by conformal mappings, Pacific J. Math. 3 (1953), 417-436.

[20] N. Mirsky, L. Sario and C. Wang, Bounded polyharmonic functions and the dimension of the manifold, J. Math. Kyoto Univ. 13 (1973), 529-535.

[21] — Parabolicity and existence of bounded or Dirichlet finite polyharmonic functions, Rend. Ist. Mat. Univ. Trieste 6 (1974), 1-9.

[22] M. Nakai and L. Sario, Completeness and function-theoretic degeneracy of Riemannian spaces, Proc. Nat. Acad. Sci. 57 (1967), 29-31.

[23] —-, Biharmonic classification of Riemannian manifolds, Bull. Amer. Math. Soc. 77 (1971), 432-436.

[24] - Quasiharmonic classification of Riemannian manifolds, Proc. Amer. Math. Soc. 31 (1972), 165-169.

[25] — Dirichlet finite biharmonic functions with Dirichlet finite Laplacians, Math. Z. 122 (1971), 203-216.

[26] - A property of biharmonic functions with Dirichlet finite Laplacians, Math. Scand. 29 (1971), 307-316.

[27] — Existence of Dirichlet finite biharmonic functions, Ann. Acad. Sci. Fenn. A. I. 532 (1973), 1-33.

[28] — Existence of bounded biharmonic functions, J. Reine Angew. Math. 259 (1973), 147-156.

[29] - Existence of bounded Dirichlet finite biharmonic functions, Ann. Acad. Sci. Fenn. A. I. 505 (1972), 1-12.

[30] - Biharmonic functions on Riemannian manifolds, Continuum Mechanics and Related Problems of Analysis, Nauka, Moscow, 1972, 329-335.

[31] L. Sario, Biharmonic and quasiharmonic functions on Riemannian manifolds, 
Duplicated lecture notes 1968-70, University of California, Los Angeles.

[32] - Quasiharmonic degeneracy of Riemannian $N$-manifolds, Kōdai Math. Sem. Rep. 26 (1974), 53-57.

[33] - Completeness and existence of bounded biharmonic functions on a Riemannian manifold, Ann. Inst. Fourier (Grenoble) 24 (1974), 311-317.

[34] — A criterion for the existence of biharmonic Green's functions, Research Announcement, Bull. Amer. Math. Soc. 80 (1974), 1183-1184. To appear in extenso in J. Austral. Math. Soc.

[35] —- Biharmonic measure, Ann. Acad. Sci. Fenn. A. I. 587 (1974), 1-18.

[36] — Biharmonic Green's functions and harmonic degeneracy, J. Math. Kyoto Univ. 15 (1975), 351-362.

[37] L. Sario and M. Nakai, Classification Theory of Riemann Surfaces, SpringerVerlag, 1970, $446 \mathrm{pp}$.

[38] L. Sario and C. Wang, The class of $(p, q)$-biharmonic functions, Pacific J. Math. 41 (1972), 799-808.

[39] — Counterexamples in the biharmonic classification of Riemannian 2-manifolds, Pacific J. Math. 50 (1974), 159-162.

[40] - Generators of the space of bounded biharmonic functions, Math. Z. 127 (1972), 273-280.

[41] — Quasiharmonic functions on the Poincaré $N$-ball, Rend. Mat. (4) 6 (1973), $1-14$.

[42] - Riemannian manifolds of dimension $N \geq 4$ without bounded biharmonic functions, J. London Math. Soc. (2) 7 (1974), 635-644.

[43] - Existence of Dirichlet finite biharmonic functions on the Poincare 3-ball, Pacific J. Math. 48 (1973), 267-274.

[44] —-, Negative quasiharmonic functions,' Tôhoku Math. J. 26 (1974), 85-93.

[45] —_, Radial quasiharmonic functions, Pacific J. Math. 46 (1973), 515-522.

[46] - Parabolicity and existence of bounded biharmonic functions, Comm. Math. Helv. 47 (1972), 341-347.

[47] - Positive harmonic functions and biharmonic degeneracy, Bull. Amer. Math. Soc. 79 (1973), 182-187.

[48] —-, Parabolicity and existence of Dirichlet finite biharmonic functions, J. London Math. Soc. 8 (1974), 145-148.

[49] —- Harmonic and biharmonic degeneracy, Kōdai Math. Sem. Rep. 25 (1973), 392-396.

[50] - Harmonic $L^{p}$-functions on Riemannian manifolds, Kōdai Math. Sem. Rep. 26 (1975), 204-209.

[51] L. Sario, C. Wang and M. Range, Biharmonic projection and decomposition, Ann. Acad. Sci. Fenn. A. I. 494 (1971), 1-14.

[52] G. Szegö, Remark on the preceding paper by Charles Loewner, Pacific J. Math. 3 (1953), 437-446.

[53] C. Wang, Biharmonic Green's function and quasiharmonic degeneracy, Math. Scand. 35 (1974), 38-42.

[54] —, Biharmonic Green's function and biharmonic degeneracy, Math. Scand. 37 (1975), 122-128.

[55] C. Wang and L. Sario, Polyharmonic classification of Riemannian manifolds, J. Math. Kyoto Univ. 12 (1972), 129-140.

University of California, Los Angeles 